\title{
Gastric floating in-situ gel as a strategy for improving anti-inflammatory activity of meloxicam
}

\author{
Mohammed Jafar ${ }^{1 *}$, Mohammed Salahuddin², Srinivasa Rao Bolla ${ }^{3}$ \\ ${ }^{1}$ Department of Pharmaceutics, College of Clinical Pharmacy, Imam Abdulrahman Bin Faisal University, Dammam, Saudi Arabia. \\ ${ }^{2}$ Department of Animal facility, Institute for Research and Medical Consultation, Imam Abdulrahman Bin Faisal University, Dammam, Saudi Arabia. \\ ${ }^{3}$ Department of Anatomy, College of Medicine, Imam Abdulrahman Bin Faisal University, Dammam, Saudi Arabia.
}

\section{ARTICLE INFO \\ Received on: 19/02/2018 \\ Accepted on: 24/09/2018 \\ Available online: 30/11/2018}

Key words:

Meloxicam, inclusion

complex, NSAID, in-situ gel, in vivo activity.

\begin{abstract}
The present study was aimed to develop gastric floating in-situ gels of meloxicam (MLX) mainly to enhance antiinflammatory activity and alleviate gastric ulceration potential of meloxicam. Ternary inclusion complex of meloxicam containing hydrox ypropyl beta cyclodextrin (HP $\beta C D$ ) and diethylamine (DEA) in 1:1:1 molar ratio was used as a chief component in the development of gastric floating in-situ gel formulations of meloxicam. Box-Behnken design was utilized to design and optimize gastric floating in-situ gels of meloxicam. Independent variables (concentrations of sodium alginate, calcium carbonate, and a ternary inclusion complex of meloxicam, respectively) were optimized in order to achieve the desired responses. The response surface plots and the possible interactions between the independent variables were analyzed using the Design Expert Software 11.0.3.0 (Stat-Ease, Inc, USA). The results showed that the optimized gastric floating in-situ gels with a short floating lag time (41 seconds), low viscosity (190 cps), and high in vitro drug release at sixth hour (77\%) was obtained using an optimized combination of calcium carbonate $(0.75 \% \mathrm{w} / \mathrm{v})$, sodium alginate $(1.25 \% \mathrm{w} / \mathrm{v})$, and MLX-HPßCD-DEA ternary complex (equivalent to $11.25 \mathrm{mg}$ of meloxicam), respectively. Moreover, the optimized gastric floating in-situ gel formulation of meloxicam ternary complex exhibited significantly ameliorated anti-inflammatory activity $[84.38 \%(p<0.05)$ at sixth hour and also showed a significant reduction in local gastric ulceration potential compared to pure meloxicam]. Thus, this gastric floating in situ gelling system can be translated for existing and established non-steroidal anti-inflammatory drugs (NSAID) as well as formulations.
\end{abstract}

\section{INTRODUCTION}

Oral liquids offer several advantages over other oral pharmaceutical formulations such as tablets, capsules, and pills but they often suffer from abrupt gastrointestinal transit. This could be a serious concern for the majority of drugs, particularly weakly acidic drugs as they are absorbed from the stomach and/or upper part of the small intestine (El-Kamel et al., 2001; Rouge et al., 1996). The gastric retention of oral solutions containing these drugs could be favorably achieved through a radical approach of the liquid insitu gelling system. These systems are polymeric gel formulations

\section{"Corresponding Author}

Mohammed Jafar, Assistant Professor, Department of Pharmaceutics, College of Clinical Pharmacy, Imam Abdul Rahman Bin Faisal University, Dammam, Saudi Arabia.E-mail:mjomar@iau.edu.sa that respond to physical or chemical signals, including $\mathrm{pH}$, ionic factor, metabolite, or temperature (Kubo et al., 2003; Miyazaki et al., 2001; Prabaharan and Mano, 2006). Numerous polymers such as pectin (Ghare and Mundada, 2017), chitosan (Belhadji et al., 2017), gellan gum (Kerdsakundee et al., 2016), xyloglucan, and xanthan gum have been investigated for this purpose. Sodium alginate is a linear block polysaccharide copolymer made of $\beta$-Dmannuronic acid $(\mathrm{M})$ and $\alpha$-L-guluronic acid $(\mathrm{G})$ residues joined by 1,4 glycosidic linkages. It undergoes gel formation by virtue of temperature change or because of the presence of cations (e.g., $\mathrm{Na}^{+}, \mathrm{K}^{+}, \mathrm{Ca}^{+2}$ ). It is a water-soluble polysaccharide. It produces a gel via formation of double helices, followed by their ionic cross-linking. Incorporation of suitable amounts of gas-forming substance like calcium carbonate to the aqueous solutions of the above polymers could make them float on the surface of the gastric fluid (Rajinikanth et al., 2007). This floating property of the gels could help in enhancing clinical response and also minimizing the 
gastric irritant effect of weakly acidic drugs by preventing direct contact with the stomach mucosa (Thanoo et al., 1993).

Over the years, substantial work mainly related to the meloxicam-cyclodextrin and/or hydrophilic excipient complex/ dispersion incorporated formulations such as tablets (Samprasit et al., 2015), suspension (Awasthi et al., 2011), suppositories (Gowthamarajan et al., 2002), and buccal patches (Jafar and Ali, 2011) has been reported in the literature. However, except our past work (Jafar et al., 2017), there is a lack of major evidences in the literature about the meloxicam ternary complex (prepared using cyclodextrins and alkali substance combination) incorporated gastric floating in-situ gels, even though this has been considered to be very beneficial from the therapeutic point of view.

Therefore, the present study was planned to develop meloxicam ternary complex incorporated gastric floating in-situ gels to improve in vivo anti-inflammatory activity and reduce local ulceration potential of meloxicam. Box-Behnken design (Box and Wilson, 1951), which gives small number of experimental runs and takes less time, and thus, offers a far more effective approach than the traditional approaches concerning statistical optimization of a pharmaceutical formulation (Alam et al., 2016) was used in the design of gastric floating in-situ gels of meloxicam-hydroxypropyl beta cyclodextrin-diethanolamine ternary complex.

\section{MATERIALS AND METHODS}

\section{Materials}

Meloxicam is purchased from the UFC Biotechnology New York (USA), Sodium alginate and calcium carbonate were purchased from the Research Lab Fine Chemicals, Mumbai (India), while other ingredients used were of analytical research grade.

\section{Preparation of gastric floating in-situ gels}

Gastric floating in-situ gels of meloxicam (MLX)hydroxypropyl beta cyclodextrin (HP $\beta C D$ )-diethylamine (DEA) ternary complex were prepared as per the reported method (Shendge et al., 2014). Weighed quantity of sodium alginate was transferred to a beaker containing half of the total volume of double distilled water, which contains $0.25 \% \mathrm{w} / \mathrm{v}$ of sodium citrate. The contents of the beaker were heated to $90^{\circ} \mathrm{C}$ with continuous stirring on a magnetic stirrer until a clear solution was obtained and then the solution was allowed to cool below $40^{\circ} \mathrm{C}$. Calculated amounts of calcium carbonate and MLX-HP $\beta C D-D E A$ ternary complex were dissolved in the second half of the double distilled water in a separate beaker and this solution was slowly added with continuous stirring to the cooled sodium alginate solution. Finally, the in-situ gelling solution obtained was stored in an amber colored glass bottle in a cool place until further investigation.

\section{Optimization}

Based on our previous published work (Jafar et al, 2018), MLX-HPßCD-DEA ternary complex (1:1:1 molar ratio) was selected as one of the factors influencing the performance of gastric floating in-situ gels of meloxicam. The other two independent variables or factors, i.e., calcium carbonate and sodium alginate were selected based on factor screening study. The Box-Behnken experimental design was employed to optimize the floating in-situ gelling solutions wherein the concentrations of calcium carbonate (A), sodium alginate (B), and MLX-HP $\beta C D$ DEA ternary complex (C) were selected as independent variables or factors. Each factor was kept as low, medium, and high levels. Floating lag time, viscosity, and percent cumulative drug release at sixth hour were taken as dependent variables or responses (Table 1). The effect of factors on the observed responses was analyzed employing Design expert version 11.0.3.0 (Stat-Ease, Inc, USA) software. Fifteen experimental runs obtained from the design with three middle points with their observed responses are depicted in Table 2. The responses were statistically analyzed by the analysis of variance (ANOVA) test method. The optimum formulation was chosen by the numerical optimization process using the desirability function. In order to assess the impact of each factor on the observed responses, the polynomial coefficients for in-situ gelling solutions were ascertained. The polynomial equation generated by this experimental design is as follows:

$$
\begin{aligned}
Y= & b_{0}+b_{1} A+b_{2} B+b_{3} C+b_{12} A B+b_{13} \mathrm{AC}+b_{23} \mathrm{BC}+b_{11} \mathrm{~A}^{2} \\
& +b_{22} \mathrm{~B}^{2}+b_{33} \mathrm{C}^{2}
\end{aligned}
$$

Where $Y$ is the response; $b_{0}$ is the intercept; $b_{1}-b_{33}$ are the regression coefficients calculated from the observed experimental values; and $A, B$, and $C$ are the coded levels of the factors. The terms $A, B$, and $C_{i}^{2}(i=1,2$, or 3$)$ constitute the interaction and quadratic terms, respectively.

\section{In-vitro evaluation of gastric floating in-situ gels of MLX- HPßCD-DEA ternary complex}

\section{Appearance}

The color and the clarity of the gastric floating in-situ gels of MLX-HP $\beta C D-D E A$ ternary complex were evaluated by the visual inspection of the solutions against a dark illuminating background.

Table 1. Variables in a Box-Behnken design for the formulation of gastric floating in-situ gels of

\begin{tabular}{|c|c|c|c|}
\hline \multirow{2}{*}{ Factors } & \multicolumn{3}{|c|}{ Level used, actual coded } \\
\hline & Low $(-1)$ & Medium (0) & High $(+1)$ \\
\hline $\mathrm{A}=$ calcium carbonate $(\%)$ & 0.25 & 0.5 & 0.75 \\
\hline $\mathrm{B}=$ sodium alginate $(\%)$ & 1.25 & 1.75 & 2.25 \\
\hline $\mathrm{C}=\mathrm{MLX}-\mathrm{HP} \beta \mathrm{CD}-\mathrm{DEA} \cong$ meloxicam $(\mathrm{mg})$ & 7.5 & 11.25 & 15 \\
\hline Dependent variables & \multicolumn{3}{|c|}{ Goals } \\
\hline $\mathrm{Y}_{1}=$ floating lag time $(\mathrm{Sec})$ & \multicolumn{3}{|c|}{ Shorten } \\
\hline $\mathrm{Y}_{2}=$ viscosity $(\mathrm{cps})$ & \multicolumn{3}{|c|}{ Decrease } \\
\hline $\mathrm{Y}_{3}=$ cumulative drug release $(\%)$ & \multicolumn{3}{|c|}{ Enhance and prolong } \\
\hline
\end{tabular}
MLX-HP $\beta C D-D E A$ ternary complex. 
Table 2. Observed response in Box-Behnken design for gastric floating in-situ gels of MLX-HP $\beta C D-D E A$ ternary complex.

\begin{tabular}{|c|c|c|c|c|c|c|}
\hline \multirow{3}{*}{ Run no. } & \multicolumn{3}{|c|}{ Variables $^{*}$} & \multicolumn{3}{|c|}{ Responses } \\
\hline & $\mathbf{A}$ & B & C & Floating lag time & Viscosity & Cumulative drug release \\
\hline & $\% w / w$ & $\% w / w$ & $\mathrm{mg}$ & (Seconds) & (cps) & $(\%)$ \\
\hline 1 & 0.25 & 1.25 & 11.25 & 340 & 1,160 & 54 \\
\hline 2 & 0.25 & 1.75 & 15 & 334 & 472 & 72 \\
\hline 3 & 0.75 & 2.25 & 11.25 & 54 & 1190 & 52 \\
\hline 4 & 0.25 & 1.75 & 7.5 & 342 & 430 & 69 \\
\hline 5 & 0.5 & 2.25 & 15 & 174 & 1,180 & 55 \\
\hline 6 & 0.5 & 1.75 & 11.25 & 170 & 440 & 61 \\
\hline 7 & 0.75 & 1.25 & 11.25 & 41 & 190 & 77 \\
\hline 8 & 0.25 & 2.25 & 11.25 & 338 & 1,150 & 59 \\
\hline 9 & 0.75 & 1.75 & 7.5 & 51 & 413 & 71 \\
\hline 10 & 0.5 & 1.75 & 11.25 & 170 & 440 & 61 \\
\hline 11 & 0.5 & 2.25 & 7.5 & 160 & 1,130 & 57 \\
\hline 12 & 0.5 & 1.25 & 15 & 160 & 210 & 77 \\
\hline 13 & 0.5 & 1.25 & 7.5 & 180 & 170 & 75 \\
\hline 14 & 0.5 & 1.75 & 11.25 & 170 & 440 & 61 \\
\hline 15 & 0.75 & 1.75 & 15 & 50 & 420 & 66 \\
\hline
\end{tabular}

\section{pH measurement}

The $\mathrm{pH}$ of gastric floating in-situ gels of MLX-HP $\beta C D-$ DEA ternary complex was measured by a calibrated digital $\mathrm{pH}$ meter (HI-2214 logging $\mathrm{pH}$ bench meter, UK) at room temperature using $30 \mathrm{ml}$ of the sample. The $\mathrm{pH}$ determination for each sample was performed in triplicate.

\section{Viscosity measurement}

The viscosity of gastric floating in-situ gels of MLXHP $\beta C D-D E A$ ternary complex was determined by a Viscometer (SV-10 Japan) at a room temperature using $30 \mathrm{ml}$ of the sample. Viscosity determination for each sample was done in triplicate.

\section{In-vitro gelation study}

In vitro gelation study was conducted on gastric floating insitu gels of MLX-HP $\beta C D-D E A$ ternary complex as described in the literature (Sharma et al., 2014). Ten milliliter of the gelling solution was transferred to $500 \mathrm{ml}$ of $0.1 \mathrm{~N} \mathrm{HCL}(\mathrm{pH} 1.2)$ in a beaker without much turbulence to prevent shattering of the formed gel. Gelling was observed in the beaker by visual inspection and the formulations based on their gelling consistency were given with different grades.

\section{In-vitro floating study}

In vitro floating study of in-situ gels of MLX-HP $\beta C D$ DEA ternary complex was conducted as per the method reported in the literature (Rajinikanth and Mishra, 2009). Five hundred milliliter of the dissolution medium ( $\mathrm{pH}$ 1.2) is taken in a dissolution flask (USP Type-II) and the temperature of the dissolution medium was maintained at $37^{\circ} \mathrm{C} \pm 0.5^{\circ} \mathrm{C}$. Ten milliliter of the solution is transferred to a Petri dish ( $4.5 \mathrm{~mm}$ internal diameter) and the Petri dish is carefully placed at the bottom of a dissolution flask without much disturbance in the flask. The time taken by the in-situ gel to come up to the surface of the medium (floating lag time) and also about how much time the gel continuously floated on the surface of the medium (duration of floating) was recorded.
In-vitro drug release study

In vitro release of meloxicam from the gastric floating insitu gels of MLX-HP $\beta C D-D E A$ ternary complex was determined in a USP XXIV rotating paddle apparatus (eight basket Dissolution Test Station, Electrolab, India) at $37^{\circ} \mathrm{C}$ using the paddle method at $50 \mathrm{rpm} /$ minute. The dissolution medium used was $500 \mathrm{ml}$ of 0.1 $\mathrm{N}$ Hydrochloric acid ( $\mathrm{pH}$ 1.2) which was prepared and degassed using a media preparator (EMP-21 DO, Electrolab, India). The insitu gel is added to a Petri dish (4.5 mm internal diameter) and the Petri dish is then transferred to the bottom of a dissolution basket without much disturbance. Five milliliter samples were withdrawn at fixed time intervals and analyzed at $362 \mathrm{~nm}$ using UV-Visible Spectrophotometer (BT-600 UK). After each withdrawal, an immediate replacement of $5 \mathrm{ml}$ fresh dissolution medium was done to maintain a sink condition. Each determination was performed in triplicate till 6 hours.

\section{In-vivo activity}

The in vivo activity experimental protocol was approved by the Institutional Review Board and Animal Ethical Committee of the Imam Abdul Rahman Bin Faisal University (formerly University of Dammam), Dammam, Kingdom of Saudi Arabia (Approval number: IRB-2014-3-199).

Animals

Wistar rats of either sex weighing 200-250 g were used in the present study. The selected animals were housed in polypropylene cages under standard laboratory conditions (temperature $25^{\circ} \mathrm{C}+$ $2^{\circ} \mathrm{C}$ ) with a cycle of 12 hours of darkness and light. The animals were fed with standard diet and water ad libitum and were fasted for at least 12 hours prior to the anti-inflammatory activity experiment.

\section{Carrageenan-induced rat hind paw edema method}

Carrageenan-induced rat hind paw edema method (Winter et al., 1968) was used to assess the anti-inflammatory activity of 
optimized gastric floating in-situ gels of MLX-HP $\beta C D-D E A$ ternary complex. Rats were divided into three groups; each group has six rats. The animals of group I received $1 \mathrm{ml}$ of control [Calcium carbonate/ sodium alginate $(0.75 \% \mathrm{w} / \mathrm{v} / 1.25 \% \mathrm{w} / \mathrm{v})]$ per oral and groups II and III received per oral Standard [Calcium carbonate/sodium alginate/ pure meloxicam $(0.75 \% \mathrm{w} / \mathrm{v} / 1.25 \% \mathrm{w} / \mathrm{v} / 11.25 \mathrm{mg})]$ and optimized gastric floating in-situ gel [Calcium carbonate/sodium alginate/ $(0.75 \% \quad \mathrm{w} / \mathrm{v} / 0.25 \% \mathrm{w} / \mathrm{v} / \mathrm{MLX}-\mathrm{HP} \beta \mathrm{CD}-\mathrm{DEA}$ ternary complex equivalent to $11.25 \mathrm{mg}$ of meloxicam)], respectively (Dose of meloxicam: $1 \mathrm{mg} / \mathrm{kg}$ body weight). After 1 hour of the test drug administration and the control treatment animals of groups I, II, and III were injected with subcutaneous injection of $0.1 \mathrm{ml}$ of $1 \% \mathrm{w} / \mathrm{v}$ solution of carrageenan (Sigma Chemical Co, St. Louis, $\mathrm{MO}$ ) in normal saline into the plantar side of the left hind paw. Plethysmograph (UGO Basile, Italy) was used to measure the rat paw volume. The paw volume was measured at $0,1,2,3,4$, and 6 hours after the injection of carrageenan.

The edema inhibitory activity was computed using the following formula:

$\%$ edema inhibition $=(1-D / C) 100$

Where,

$D$-represents the percentage difference in increased paw volume after the administration of test drugs to the rats.

$C$-represents the percentage difference of increased volume in the control groups.

\section{Ulcerogenic study}

For the gastric mucosal damage studies, rats were not fed for 24 hours but the water was made available to them. The rats of the control group were given normal saline, the standard drug group were given $15 \mathrm{mg} / \mathrm{kg}$ body weight of meloxicam standard drug, and the formulation group were given $15 \mathrm{mg} / \mathrm{kg}$ body weight of optimized gastric floating in-situ gel of meloxicam. All the rats were euthanized 4 hours after the drug administration and stomachs were excised and were cut open along the greater curvature from the oesophagus to the pylorus. Stomachs were placed on a corkboard and pinned, the ulcer score was done by examining under the dissection microscope. A blind observer unaware of the experimental groups was requested to assess the mucosal damage.Scoring sheet (Mishra and Vijay Kumar, 2006) containing the following rubrics was used for scoring

$$
\begin{aligned}
& 0 \text { - no injury or bleeding } \\
& 1 \text { - slight injury (two to three dots) } \\
& 2 \text { - severe injury (hemorrhagic streaks, five to six dots) } \\
& 3 \text { - very severe injury (many lined injuries) } \\
& 4 \text { - mucosa full of lesions }
\end{aligned}
$$

\section{Statistical analysis}

For ulcer scoring study, we used SPSS version 23. The data are expressed as mean $\pm \mathrm{SE}$. One ANOVA was performed to compare between groups followed by Tukey's post hoc test. $p$ value $<0.05$ was considered significant.

\section{RESULTS AND DISCUSSION}

\section{Optimization}

Fifteen gastric floating in-situ gels of MLX-HP $\beta C D-D E A$ ternary complex were developed according to the experimental design and characterized by different responses such as floating lag time, viscosity, and drug release. The mathematical correlations were set up and coefficients of the second order polynomial equations were derived using multiple linear regression analysis for a floating lag time, viscosity, and drug release were found to be quadratic in nature with interaction terms. The coefficients of the polynomials fit well to the data, with the values of $R^{2}$ ranging between 0.4373 and 0.9957 ( $p<0.005$ in all cases). The three dependent values ranged from 41 to 342 seconds, $170-1,190 \mathrm{cps}$, and $52 \%-77 \%$ for a floating lag time, viscosity, and drug release, respectively. A positive value in polynomial equations corresponds to an effect that favors the optimization, whereas a negative value represents an inverse relationship between the factor and the response. The polynomial equations derived from the statistical analysis of the results are given in Table 3 . Where $A, B$, and $C$ correspond to the coded values of the calcium carbonate, sodium alginate, and meloxicam, respectively. The effect of calcium carbonate on floating lag time is comparatively more significant than the effect of sodium alginate. Other independent variable meloxicam has not shown any significant change in the floating lag time of in-situ gelling solutions. All three independent variables, namely calcium carbonate, sodium alginate, and meloxicam individually and also in combinations showed the positive effect on viscosity. But the effect of the said variables is negative on drug release. This could be due to the sol to gel transformation of the formulations in an acidic medium.

All the responses studied for 15 in-situ gel formulations were collectively fitted to various models using Design expert version 11.0.3.0 (Stat-Ease, Inc, USA). The best-fitted model of the three factors and their comparative values of $R^{2}$, predicted $R^{2}$, adjusted $R^{2}$, standard deviation (SD), and $\% \mathrm{CV}$ are given in Table 3. The "predicted $R^{2}$ " was more or less in accordance with the "adjusted $R^{2}$ " values (Fig. 1). The 3D response surface graphs presenting the interaction effects of the factors on the responses are illustrated in Figure 2. The fitting results showed that the optimized in-situ gels of MLX-HP $\beta C D$-DEA ternary complex with short floating lag time (41 seconds), low viscosity (190 cps), and high drug release at the sixth hour (77\%) was obtained using an optimized combination of calcium carbonate $(0.75 \% \mathrm{w} / \mathrm{v})$, sodium alginate $(1.25 \% \mathrm{w} / \mathrm{v})$, and meloxicam content (11.25 mg), respectively. All the response surfaces were best fitted with quadratic polynomial models and are capable of predicting the interaction effects as well. Finally, the model was analyzed for ANOVA $(p<0.005)$, which disclosed that the model terms for main effects and interaction effects were highly significant.

\section{In-vitro evaluation of gastric floating in-situ gels}

All 15 in-situ gels of MLX-HPßCD-DEA ternary complex were clear, viscous, and pale yellow in color. The $\mathrm{pH}$ of the solutions was in the range of 6.8-7.5. The in-situ gelling solutions containing $0.5 \%$ and above amount of calcium carbonate exhibited desired in vitro gelation property and also showed a long duration of floating on the surface of an acidic medium ( $\mathrm{pH}$ 1.2). This could be due to the free availability of a sufficient number of calcium ions and carbon dioxide content from the calcium carbonate in an acidic medium to boost the gelling potential of sodium alginate and also induce floating 
Table 3. Model summary statistics given by a Box-Behnken design.

\begin{tabular}{|c|c|c|c|c|c|}
\hline Model & $R^{2}$ & Adjusted $R^{2}$ & Predicted $R^{2}$ & SD & $\% \mathrm{CV}$ \\
\hline \multicolumn{6}{|c|}{ Floating lag time } \\
\hline Linear & 0.9843 & 0.9801 & 0.9691 & 15.58 & \multirow[t]{3}{*}{1.65} \\
\hline $2 \mathrm{FI}$ & 0.9864 & 0.9762 & 0.9388 & 17.00 & \\
\hline Quadratic & 0.9997 & 0.9993 & 0.9957 & 3.01 & \\
\hline \multicolumn{6}{|l|}{ Viscosity } \\
\hline Linear & 0.5271 & 0.3981 & 0.0342 & 311.95 & \multirow[t]{3}{*}{32.06} \\
\hline $2 \mathrm{FI}$ & 0.6399 & 0.3698 & 0.7200 & 319.20 & \\
\hline Quadratic & 0.9102 & 0.7485 & 0.4373 & 201.66 & \\
\hline \multicolumn{6}{|c|}{ Cumulative $\%$ drug release (sixth hour) } \\
\hline Linear & 0.4576 & 0.3097 & 0.1265 & 7.10 & \multirow[t]{3}{*}{6.95} \\
\hline $2 \mathrm{FI}$ & 0.6970 & 0.4697 & 0.4667 & 6.23 & \\
\hline Quadratic & 0.9018 & 0.7251 & 0.5707 & 4.48 & \\
\hline
\end{tabular}

characters in it. These findings are consistent with the reported results (Rajinikanth et al., 2007).

The in-situ gels demonstrated a considerable increase in viscosity with increasing amount of sodium alginate. It was attributed to an increasing chain interaction with sodium alginate concentration. Similarly, an increase in the amount of calcium carbonate also increases the viscosity of the in-situ gels at all three sodium alginate percentage. It could be due to the high concentration of finely dispersed particles of calcium carbonate in the gelling solution. On the other hand, meloxicam has not contributed much in increasing the viscosity of in-situ gels as it was in its freely soluble ternary complex form with Hydroxypropyl $\beta$-Cyclodextrin and diethanolamine.

A marked decline in the rate and extent of in-vitro drug release was noted with the increase in sodium alginate concentration in in-situ gels (Fig. 3). It is attributed to the high density of the system and also to the increase in the drug's diffusion path length. In order to study the drug release mechanism, the in vitro release data obtained were fitted to various kinetic equations. The release model of optimized in-situ gel formulation followed Matrix (Higuchi matrix) kinetics.

\section{In-vivo anti-inflammatory activity}

The optimized floating in-situ gel of MLX-HP $\beta C D-D E A$ ternary complex has showed significant inhibition of carrageenaninduced rat paw edema from 2 to 6 hours in rats following oral administration, as compared with the control and standard group of animals. The highest percentage of inhibition of the standard drug pure meloxicam was found to be $59.37 \%(p<0.05)$, whereas the optimized floating in-situ gel of MLX-HP $\beta C D$-DEA ternary complex exhibited the maximum percentage of inhibition of paw edema at 6 hours is $84.38 \%(p<0.05)$ (Table 4$)$, even though the standard drug has exhibited the significant inhibition of paw edema but the optimized gastric floating in-situ gel has been found to be more significant $(p<0.05)$ paw edema inhibition from 2 hours to till 6 hours as compared to the control and standard group of animals.

\section{Ulcerogenic study}

The optimized gastric floating in-situ gel of meloxicam ternary complex and standard meloxicam showed significant ulcerogenic potential compared to control in rats treated with standard meloxicam/optimized formulation. The optimized formulation showed less ulcerogenic potential as compared to standard meloxicam (Fig. 4). These results clearly indicate that the optimized formulation could protect the gastric mucosa from injury (Fig. 5). Reduced gastric ulceration of the optimized formulation as compared with the standard could be due to the slow release of meloxicam from the in-situ gels (El-Kamel et al., 2001).

\section{CONCLUSION}

The optimized gastric floating in-situ gel of MeloxicamHydroxypropyl- $\beta$-Cyclodextrin-Diethanolamine ternary complex demonstrated a desired gelling and floating property with the prolonged and acceptable amount of drug release in an acidic medium. Improved anti-inflammatory activity and reduced gastric ulceration of the optimized formulation as compared to the standard could be due to the use of a ternary complex of meloxicam in the formulation and prolonged release of meloxicam from the gastric floating in-situ gel, respectively. Thus, this gastric floating insitu gelling system can be translated for existing and established NSAID as well as formulations.

\section{ACKNOWLEDGMENTS}

We sincerely thank the Deanship of Scientific research (DSR) Imam Abdul Rahman Bin Faisal University, Dammam for the financial support and we also thank the Dean of Institute for Research and Medical Consultation (IRMC), Imam Abdul Rahman Bin Faisal University, Dammam for providing animal experimentation facilities. We extend our sincere thanks to the Dean of the College of Clinical Pharmacy Imam Abdul Rahman Bin Faisal University, Dammam for providing necessary laboratory facilities for this research. 
Design-Expert] Software

Floating lag time

Color points by value of Floating lag time:

$41 \square 342$

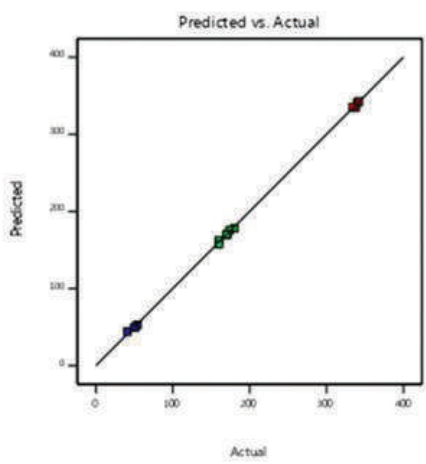

Design-Expert@ Software

Viscosity

Color points by value of Viscosity:

$170 \square 1190$

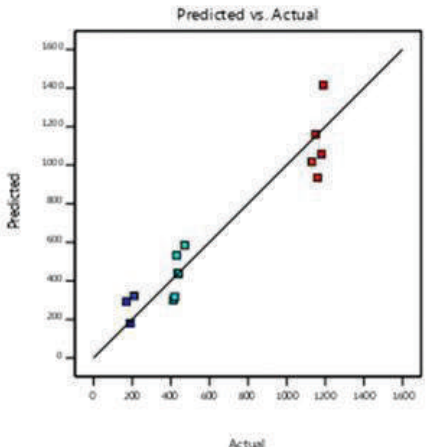

Predicted vs. Actual

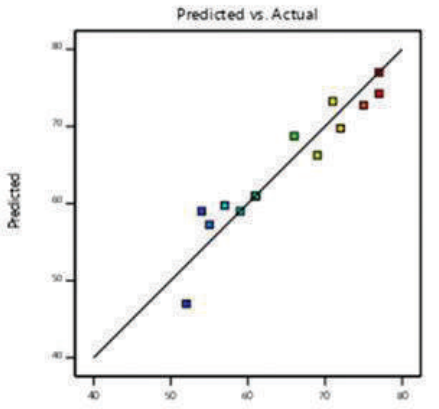

Design-Expert@ Software

Cumulative drug release

Color points by value of Cumulative drug release:

$52 \square 77$

Figure 1. Actual $v s$ Predicted values obtained from design expert software for gastric floating in-situ gels of MLX-HP $\beta C D-D E A$ ternary complex.

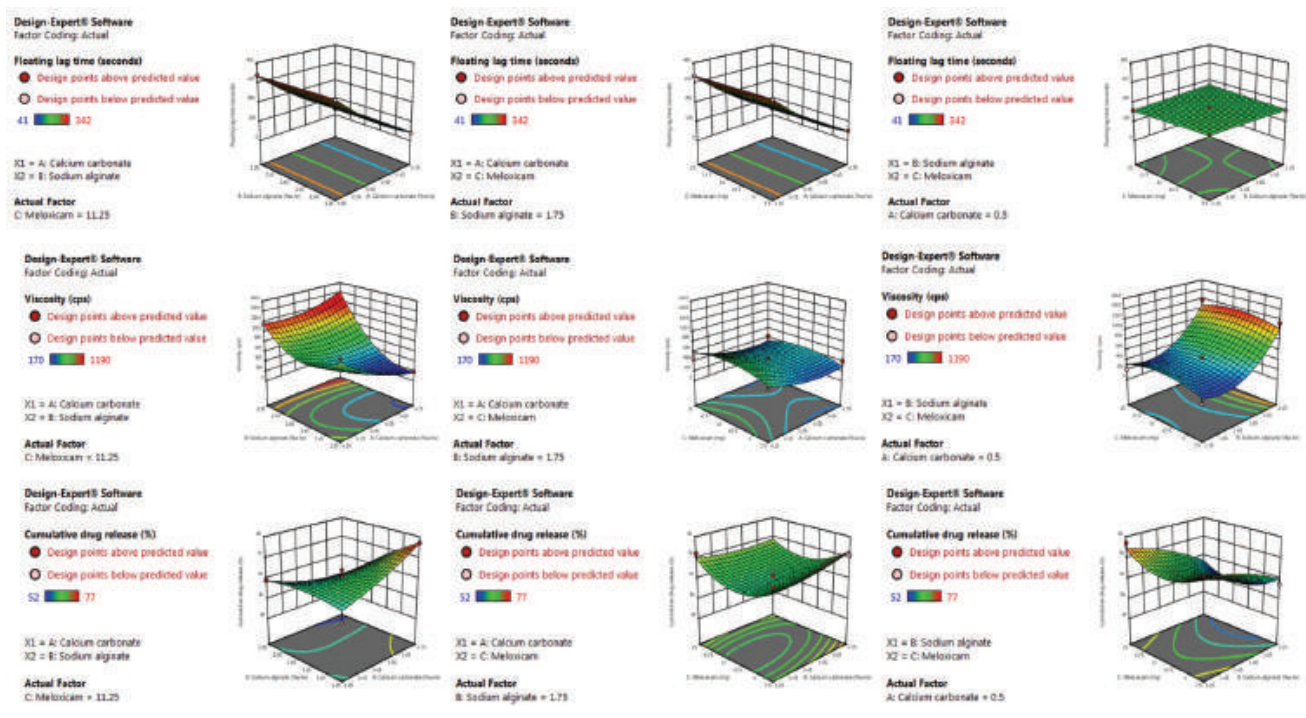

Figure 2. 3D graphs of independent variables floating lag time, viscosity, and cumulative drug release obtained from design expert software for gastric floating in-situ gels of MLX-HP $\beta C D-D E A$ ternary complex. 


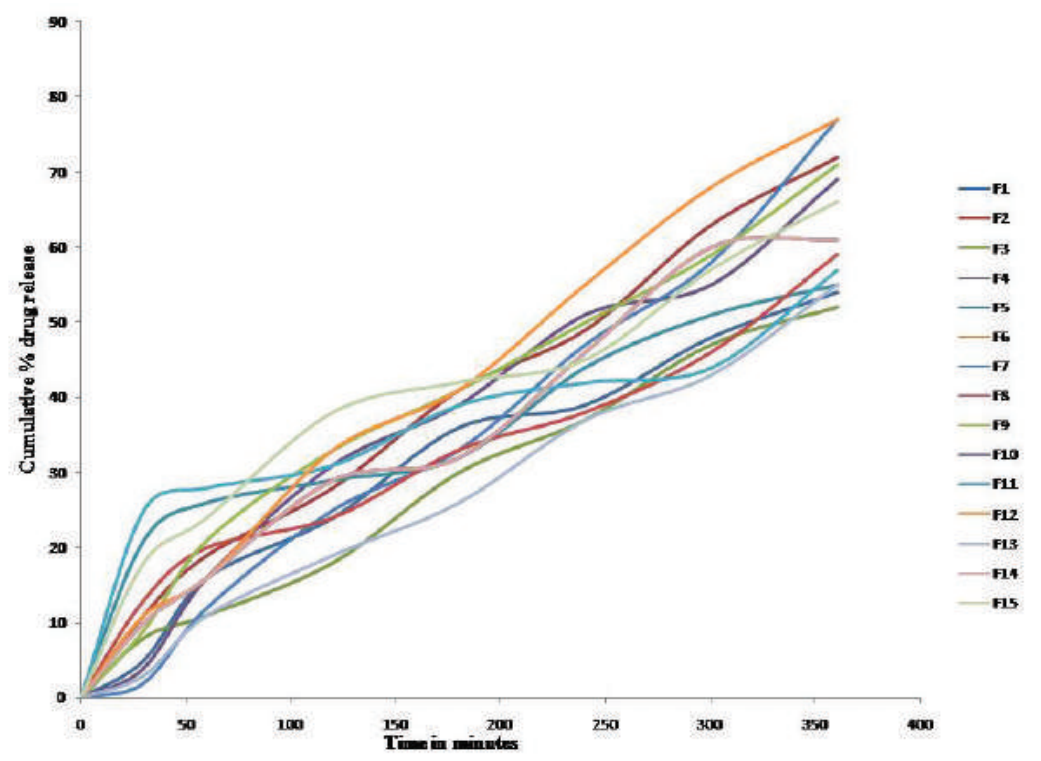

Figure 3. In-vitro drug release profile of gastric floating in-situ gels of MLX-HP $\beta C D-D E A$ ternary complex.

Table 4. Anti-inflammatory activity of optimized gastric floating in-situ gels of MLX-HP $\beta C D-D E A$ ternary complex.

\begin{tabular}{|c|c|c|c|c|c|c|c|c|c|}
\hline \multirow[b]{2}{*}{ Group } & \multirow[b]{2}{*}{ Treatment } & \multirow[b]{2}{*}{$\begin{array}{l}\text { Initial paw } \\
\text { volume }\end{array}$} & \multicolumn{7}{|c|}{ Paw volume (ml) } \\
\hline & & & 1 hour & 2 hours & 3 hours & 4 hours & 5 hours & 6 hours & $\begin{array}{l}\text { Edema inhibition } \\
\text { at sixth hour } \\
(\%)\end{array}$ \\
\hline I & Control & $1.17 \pm 0.06$ & $1.22 \pm 0.02$ & $1.47 \pm 0.07$ & $1.65 \pm 0.07$ & $1.67 \pm 0.02$ & $1.92 \pm 0.04$ & $2.18 \pm 0.06$ & --- \\
\hline II & Standard & $1.07 \pm 0.09$ & $1.18 \pm 0.04$ & $1.30 \pm 0.03^{*}$ & $1.39 \pm 0.05^{*}$ & $1.42 \pm 0.01 *$ & $1.48 \pm 0.05^{*}$ & $1.57 \pm 0.01 *$ & 59.37 \\
\hline III & Optimized formulation & $1.04 \pm 0.02$ & $1.17 \pm 0.05$ & $1.20 \pm 0.01 *$ & $1.19 \pm 0.02 *$ & $1.32 \pm 0.06^{*}$ & $1.35 \pm 0.03^{*}$ & $1.32 \pm 0.04 *$ & 84.38 \\
\hline
\end{tabular}

Values are presented as the mean \pm SEM, $n=6$ in each group; one-way ANOVA followed by multiple Tukey's comparison test. ${ }^{*} p<0.05$, as compared to the control group ANOVA $=$ analysis of variance; SEM $=$ standard error of the mean.

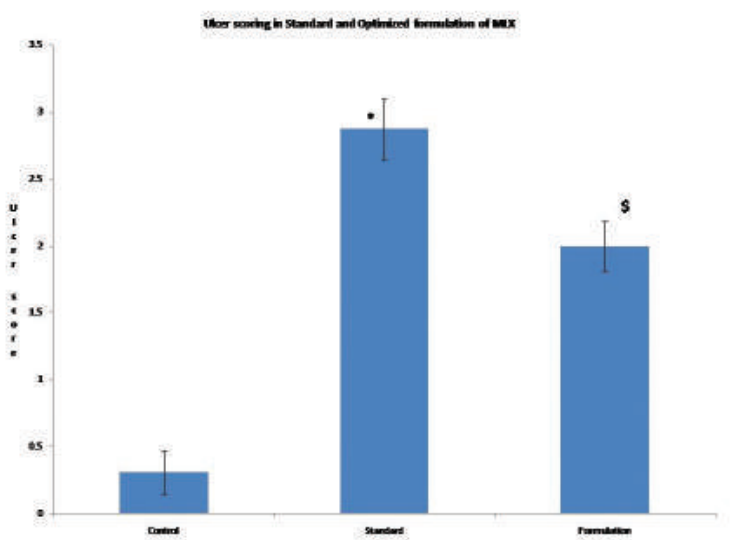

Figure 4. Ulcerogenic potential of pure meloxicam and optimized gastric floating in-situ gels of MLX-HP $\beta C D-D E A$ ternary complex.

*-Significant compared to control, \$- significant compared to standard drug group.

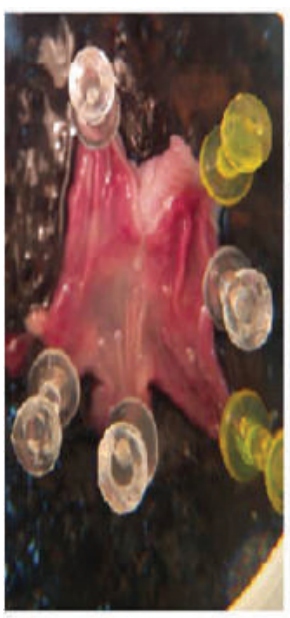

Control

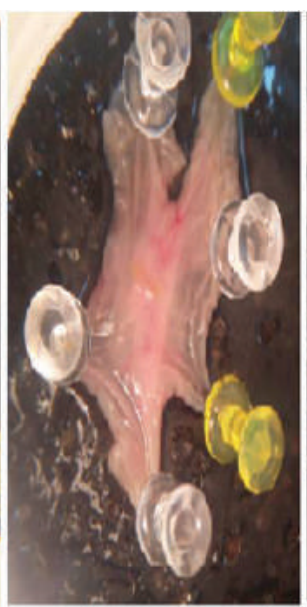

StandardMeloxican

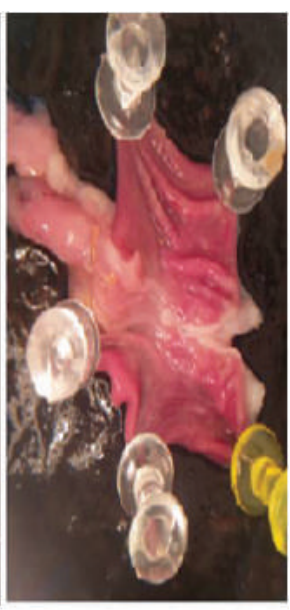

Optimized tormulation
Figure 5. Representative stomachs of rats treated with pure meloxicam and optimized gastric floating in-situ gels of MLX-HPßCD-DEA ternary complex. 


\section{DECLARATION OF INTEREST}

This work was supported by Deanship of Scientific research (DSR), Imam Abdul Rahman Bin Faisal University, Dammam, Saudi Arabia under research group project grant number: 2014299.

\section{REFERENCES}

Alam S, Aslam M, Khan A, Imam SS, Aqil M, Sultana Y, Ali A. Nanostructured lipid carriers of pioglitazone for transdermal application: from experimental design to bioactivity detail. Drug Deliv, 2016; 23(2):601-9.

Awasthi SS, Kumar TG, Manisha P, Preeti Y, Kumar SS. Development of meloxicam formulations utilizing ternary complexation for solubility enhancement. Pakistan J Pharm Sci, 2011; 24(4):533-8.

Belhadji L, HadjSadok A, Moulai-Mostefa N. Design and characterization of calcium-free in-situ gel formulation based on sodium alginate and chitosan. Drug Develop Indus Pharm, 2017; 44(4):662-9.

Box GEP, Wilson KB. On the experimental attainment of multifactorial conditions. J Royal Statis Soc, 1951; 13:1-12.

El-Kamel AH, Sokar MS, Al-Gamal SS, Naggar VF. Preparation and evaluation of ketoprofen floating oral delivery system. Int J Pharm, $2001 ; 220: 13-21$.

Ghare JL, Mundada AS. Evaluation of novel polymer in the development of floating in situ gelling system. J Appl Pharm Sci, 2017; 9:238.

Gowthamarajan K, Giriraj Kulkarni T, Venkateswaran G, Samanta MK, Suresh B. Formulation and dissolution properties of meloxicam solid dispersion incorporated suppositories. Indian J Pharm Sci, 2002; 64(6):525-8.

Jafar M, Ali S. Studies on meloxicam solid dispersion incorporated buccal patches. Int Res J Pharm, 2011; 2(5):220-7.

Jafar M, Salahuddin M, Kayed TS, Ahmad N, Al-Eid HA, Al-Qarros AH. Buoyant in situ gels of meloxicam-[beta]-cyclodextrintriethanolamine ternary complex for oral delivery; from a box-behnken experimental design to in vivo activity detail. Asian J Chem, 2017; 29(6):1275-84.

Jafar M, Salahuddin M, Kayed TS, Ahmad N, Al-Eid HA, Al-Qarross AH. Solid state analysis and in-vitro dissolution behavior of meloxicam-hydroxy propyl beta cyclodextrin-ethanolamines ternary complexes. Int J Pharm Qual Assurance, 2018; 9(1):80-6.

Kerdsakundee N, Wiwattanapatapee R, Mahattanadul S. Floating gellan gum-based in situ gels containing curcumin for specific delivery to the stomach. Thai J Pharm Sci, 2016; 40(Suppl):33-6.

Kubo W, Miyazaki S, Attwood D. Oral sustained delivery of paracetamol from in situ-gelling gellan and sodium alginate formulations. Int J Pharm, 2003; 258:55-64.
Mishra DN, Vijay Kumar SG. Investigations on analgesic, antiinflammatory and ulcerogenic potential of meloxicam solid dispersion prepared with skimmed milk. Yakugaku Zasshi, 2006; 126(7):495-8.

Miyazaki S, Kawasaki N, Kubo W, Endo K, Attwood D. Comparison of in situ gelling formulations for the oral delivery of cimetidine. Int J Pharm, 2001; 220(1-2):161-8.

Prabaharan M, Mano JF. Stimuli-responsive hydrogels based on polysaccharides incorporated with thermo-responsive polymers as novel biomaterials. Macromol Biosci, 2006; 6(12):991-1008.

Rajinikanth PS, Balasubramaniam J, Mishra B. Development and evaluation of a novel floating in situ gelling system of amoxicillin for eradication of Helicobacter pylori. Int J Pharm, 2007; 335(1-2):114-22.

Rajinikanth PS, Mishra B. Stomach-site specific drug delivery system of clarithromycin for eradication of Helicobacter pylori. Chem Pharma Bull, 2009; 57(10):1068-75.

Rouge N, Buri P, Doelkar E. Drug absorption sites in the gastrointestinal tract and dosage forms for site-specific delivery. Int J Pharm, 1996; 136(1-2):117-39.

Samprasit W, Akkaramongkolporn P, Ngawhirunpat T, Rojanarata T, Opanasopit P. Formulation and evaluation of meloxicam oral disintegrating tablet with dissolution enhanced by combination of cyclodextrin and ion exchange resins. Drug Develop Indus Pharm, 2015; 41(6):1006-16.

Sharma A, Sharma J, Kaur R, Saini V. Development and characterization of in-situ oral gel of spiramycin. BioMed Res Int, 2014; 876182.

Shendge RS, Jamdhade AA, Pande VV. Novel strategy in controlled gastroretentive drug delivery: in-situ floating gel. Int J Drug Delivery, 2014; 6:230-43.

Thanoo BC, Sunny MC, Jayakrishnan A. Oral sustained-release drug delivery systems using polycarbonate microspheres capable of floating on the gastric fluid. J Pharm Pharmacol, 1993; 45(1):21-4.

Winter CA, Risley EA, Silber RH. Antiinflammatory activity of indomethacin and plasma corticosterone in rats. J Pharmacol Exp Ther, $1968 ; 162: 196-201$.

\section{How to cite this article:}

Jafar M, Salahuddin M, Bolla SR. Gastric floating in-situ gel as a strategy for improving anti-inflammatory activity of meloxicam. J App Pharm Sci, 2018; 8(11): 095-102. 